\title{
Beam quality improvement of high-power semiconductor lasers using laterally inhomogeneous waveguides
}

Cite as: Appl. Phys. Lett. 113, 221107 (2018); https://doi.org/10.1063/1.5054645

Submitted: 01 September 2018 . Accepted: 12 November 2018 . Published Online: 30 November 2018

M. J. Miah (D) S. Strohmaier, C. Urban, and D. Bimberg
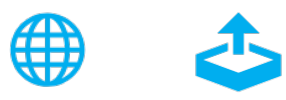

View Online

Export Citation

\section{ARTICLES YOU MAY BE INTERESTED IN}

$1.5 \mu \mathrm{m}$ quantum-dot diode lasers directly grown on CMOS-standard (001) silicon

Applied Physics Letters 113, 221103 (2018); https://doi.org/10.1063/1.5055803

All group-IV SiGeSn/GeSn/SiGeSn QW laser on Si operating up to $90 \mathrm{~K}$

Applied Physics Letters 113, 221104 (2018); https://doi.org/10.1063/1.5052563

Integration of quantum dots with lithium niobate photonics

Applied Physics Letters 113, 221102 (2018); https://doi.org/10.1063/1.5054865

\section{Lock-in Amplifiers up to $600 \mathrm{MHz}$}
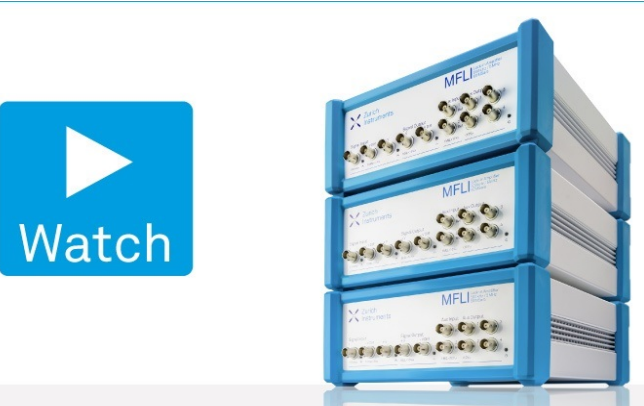


\title{
Beam quality improvement of high-power semiconductor lasers using laterally inhomogeneous waveguides
}

\author{
M. J. Miah, ${ }^{1,2, a)}$ S. Strohmaier, ${ }^{2}$ G. Urban, ${ }^{2}$ and D. Bimberg ${ }^{1,3}$ \\ ${ }_{1}^{1}$ Institut für Festkörperphysik, Technische Universität Berlin, Hardenbergstr. 36, 10623 Berlin, Germany \\ ${ }^{2}$ TRUMPF Laser GmbH, Niederlassung Berlin, Volmerstr. 10, 12489 Berlin, Germany \\ 3 "Bimberg Chinese-German Center for Green Photonics," Chinese Academy of Sciences at CIOMP, \\ Changchun 13033, China
}

(Received 1 September 2018; accepted 12 November 2018; published online 30 November 2018)

\begin{abstract}
High-brightness vertical broad-area edge-emitting (HiBBEE) semiconductor lasers in the $1060 \mathrm{~nm}$ wavelength range with excellent beam quality in both lateral and vertical directions are presented. An approach to modify the thresholds of the transverse lateral modes of ridge-waveguide (RW) lasers is investigated. It has been experimentally shown that inhomogeneities in both sides of the ridges increase optical losses of the higher-order lateral modes as compared to the fundamental mode. The resulting enhancement in the contrast of the optical losses favors the emission of the fundamental mode and improves the beam quality. Reference RW HiBBEE lasers with a $15 \mu$ m wide conventional ridge and a $2.0 \mathrm{~mm}$ long cavity provide laterally multi-lateral mode emission which is typical for RW lasers with such wide and homogeneous ridges. On the other hand, RW HiBBEE lasers with triangular-shaped corrugations in both sides of $15 \mu \mathrm{m}$ wide ridges provide single-lateral mode emission across a wide current range and improve the lateral $\mathrm{M}^{2}$ factor by more than a factor of 2 in the investigated current range. The corrugated RW HiBBEE lasers provide an almost 2 times higher brightness than the reference RW lasers. Published by AIP Publishing.
\end{abstract}

https://doi.org/10.1063/1.5054645

Applications of photonic systems in material processing, ${ }^{1,2}$ medical, ${ }^{3}$ or automotive markets ${ }^{4}$ are rapidly growing. In many cases, high-power and still better, high-brightness semiconductor lasers are becoming the strategic and often enabling components. For example, cutting stents based on laser technology typically requires very good beam quality. This cannot be provided by standard broad area emitters typically emitting more than ten times diffraction limited or worse beam quality. Due to their large wall-plug efficiency, small footprint, and low cost, semiconductor lasers are becoming more attractive for these applications and might eventually also replace the existing widely used solid state and fiber lasers. However, congenital and thus omnipresent shortcomings, such as large vertical beam divergence, the elliptic beam profile, and low output power of conventional edge-emitting semiconductor lasers (EESLs), have hindered some potential breakthroughs and market penetration in important fields and have led to large module cost in particular for 1D and 2D arrays.

Catastrophic optical mirror damage ${ }^{5}$ (COMD) is one of the major factors other than thermal effects, limiting the output power of conventional EESLs. The high optical power density above $10-20 \mathrm{MW} \mathrm{cm}^{-2}$ at the laser facets already triggers COMD. The small dimensions of high-brightness ridge-waveguide (RW) EESLs, a sub- $\mu \mathrm{m}$ sized vertical waveguide and lateral dimensions being only a few microns, result in very high power density at the facets. The thin vertical waveguide also adversely yields large vertical beam divergences $\theta_{\text {vert }}$ typically of $30^{\circ}-60^{\circ}$ full width at half maximum (FWHM).

a)jarez.miah@tu-berlin.de
A variety of laser structures ${ }^{6-12}$ have been developed to narrow $\theta_{\text {vert }}$ down to $<10^{\circ}$ and increase the output power. All these approaches rely on extension of the optical field across the vertical direction aiming to simultaneously reduce the facet load and shrink the vertical beam divergence. Nevertheless, single-transverse mode power $P_{\mathrm{SM}}$, critical for high brightness applications, hardly reached more than a few hundreds of milliwatts for most structures. Only lasers with a large optical cavity and photonic band crystal waveguide demonstrated $P_{\mathrm{SM}}>1 \mathrm{~W}$ under continuous-wave (CW) operation and delivered up to $1.9 \mathrm{~W}$ in the 1060 -nm wavelength range..$^{8,11}$ In both cases, $\theta_{\text {vert }}$ was narrowed down to $14^{\circ}$ FWHM. Further simultaneous reduction of $\theta_{\text {vert }}$ and increase in $P_{\mathrm{SM}}$ seem to be very difficult to achieve without other ideas. $^{13}$ Recently, a promising approach, namely, highbrightness vertical broad-area edge-emitting (HiBBEE) laser structures, has been proposed ${ }^{14}$ and implemented. ${ }^{15}$ The first lasers already provided a record single-transverse mode power of $2.2 \mathrm{~W}$ with a perfectly circular beam profile and a divergence of only $10^{\circ}$ in both transverse lateral and vertical directions.

In contrast to the above-mentioned techniques, which are based on vertical field extension, field expansion in the lateral direction is assumed to be another remedy to reduce the power density at the laser facets. Typical high-power EESLs have wide ridges up to several hundreds of microns. EESLs with such ridges, being perfectly parallel, allow a large amount of current to be injected and thus high output power. However, at the same time, the lasers emit a multitude of lateral modes deteriorating the beam quality in the lateral direction and prohibiting high brightness together with dynamic filamentation. Using quantum dots as active 
gain media, filamentation can be largely eliminated. ${ }^{16,17} \mathrm{~A}$ different type of laser, so called tapered laser, ${ }^{18}$ combines a separate tapered section and a narrow homogeneous ridge section. The narrow ridge section filters out the higher order lateral modes while guiding only the fundamental mode. Tightly guided optical fields in the ridge waveguide region are expanded in the tapered section. Thus, a larger output power is achievable by reducing the facet load together with a good beam quality. However, laser beams of tapered lasers suffer from a very large astigmatism of several hundred microns, ${ }^{19}$ being additionally strongly current-dependent, introducing extra burden and cost to the optical systems for real world applications.

Here, we present an alternative approach to increase single-transverse mode power and hence brightness of RW lasers. The concept is based on increasing the difference of the thresholds for lasing of the fundamental and higher order modes in RW lasers with a wide ridge, thus supporting the fundamental lateral mode. An additional loss is introduced for the higher order modes by incorporating triangularshaped corrugations in both sides of the ridges. To study the effects, we chose the HiBBEE structure reported previously. ${ }^{15}$ The resulting corrugated RW HiBBEE lasers show an improvement of the lateral beam quality by more than a factor of 2 and nearly twice larger brightness than reference RW HiBBEE lasers with similar ridge dimensions without corrugations.

The details of the vertical laser structure and the working principle of the HiBBEE lasers can be found elsewhere. ${ }^{14,15}$ The HiBBEE structures investigated here consist of a vastly asymmetric vertical waveguide with a $300 \mathrm{~nm}$ thick highly pdoped GaAs contact layer, a $1 \mu \mathrm{m}$ thick p-doped AlGaAs layer, an undoped active region, and an approximately $11 \mu \mathrm{m}$ thick n-doped AlGaAs layer sequence grown on a (100) oriented n-doped substrate. The active region contains 4 compressively strained InGaAs quantum wells separated by GaAsP barrier layers and is optimized for an efficient emission in the wavelength range of $1060 \mathrm{~nm}$. The n-doped part of the structure contains 9 pairs of aperiodic AlGaAs layers with alternating $\mathrm{Al}$ contents. The thicknesses of the individual layers and doping profile of the layer structure are designed to favor fundamental mode emission along the vertical direction. The HiBBEE lasers provide single vertical mode emission with a FWHM beam divergence of $<10^{\circ}$ as expected from the design. ${ }^{15}$

For this study, the structure is processed into corrugated RW lasers with triangular-shaped corrugations. For a comparative study, reference RW lasers with typical homogeneous ridges are also processed. The reference RW lasers have a $15 \mu \mathrm{m}$ wide ridge. As we reported in Ref. $15,8 \mu \mathrm{m}$ wide $\mathrm{RW}$ lasers from the same HiBBEE waveguide still provide single lateral mode emission up to the maximum drive current investigated, and we assumed that a ridge as wide as $10 \mu \mathrm{m}$ could yield single lateral mode emission up to a large operating current. To investigate the effect of corrugations on the beam quality of RW lasers and to ensure a multi-lateral mode emission from the reference lasers, we have chosen a ridge width as large as $15 \mu \mathrm{m}$ for this study. Figure 1(a) shows an image of the top of a fully processed reference RW HiBBEE laser. The lasers have additional $10 \mu \mathrm{m}$ wide trenches on both

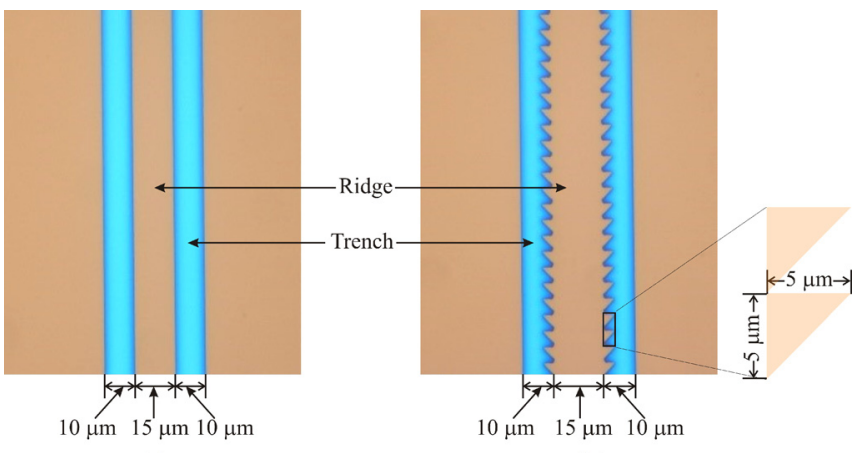

(a)

(b)

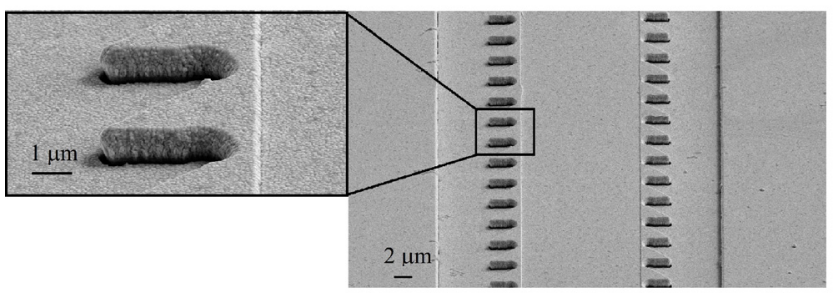

(c)

FIG. 1. Image of (a) a reference RW HiBBEE laser with a typical homogeneous ridge and (b) a corrugated RW HiBBEE laser with a triangular-shaped corrugation in both sides of the ridge. Dimensions are indicated in the figure and are not to scale. (c) Scanning electron microscopy image of the corrugated laser.

sides of the ridge. In the trenches, the layers are etched down by $610 \mathrm{~nm}$ leaving approximately $690 \mathrm{~nm}$ of the p-doped layer above the active region.

Thresholds of the fundamental and higher order lateral modes are nearly identical in RW lasers with such wide ridges, which leads to multi-lateral mode emission. ${ }^{20}$ The number of lasing modes is controlled by the width of the ridge.

Figures 1(b) and 1(c) present our corrugated RW laser with triangular-shaped inhomogeneities on both sides of the $15 \mu \mathrm{m}$ wide ridge. Additional isosceles triangles with $5 \mu \mathrm{m}$ long equal sides have been introduced at a period of $5 \mu \mathrm{m}$ throughout the whole longitudinal direction. A theoretical study $^{20}$ shows that by the introduction of such corrugations along the longitudinal direction of a RW originally supporting multiple lateral modes, a discrimination is established among the lasing thresholds of the fundamental and higher order modes. The discrimination is caused by additional scattering loss experienced by the higher order modes which are located away from the central region of the ridges.

Figure 2 depicts output power and power conversion efficiency as a function of drive current of reference and corrugated RW HiBBEE lasers in $\mathrm{CW}$ mode at $T=20^{\circ} \mathrm{C}$. Both the lasers have a $2.0 \mathrm{~mm}$ long cavity. The facets of the lasers are not passivated and are coated with high- and antireflection coatings with reflectivities of nearly $95 \%$ and $1 \%$, respectively. For heat removal in $\mathrm{CW}$ operation, the lasers are mounted on a $\mathrm{CuW}$ heat spreader and a $\mathrm{Cu}$ mount.

The reference RW laser shows a threshold current of $0.29 \mathrm{~A}$ and a differential quantum efficiency of $67 \%$. The maximum power conversion efficiency is $29 \%$. The corrugated laser demonstrates a larger threshold current of $0.33 \mathrm{~A}$ and a reduced differential quantum efficiency of $51 \%$. The reason is attributed to the losses experienced by the higher order modes due to scattering at the inhomogeneities while 


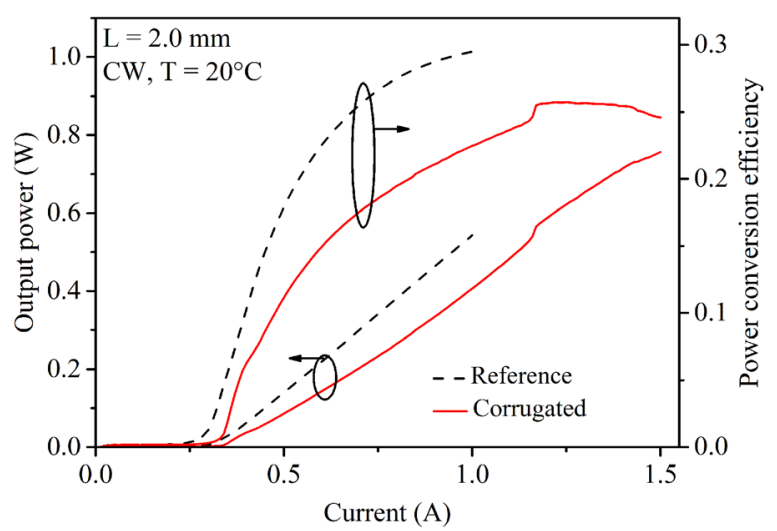

FIG. 2. Output power (left) and power conversion efficiency (right) as a function of drive current of a reference RW laser (black, dashed line) and a corrugated RW laser (red, solid line) with triangular-shaped inhomogeneities as shown in Fig. 1. The measurements are performed in $\mathrm{CW}$ mode at $T=20^{\circ} \mathrm{C}$. Both lasers have a $2.0 \mathrm{~mm}$ long cavity.

propagating along the longitudinal direction. The laser yields single-transverse mode emission up to 1.2 A drive current (as discussed later). The kink in the low current around $0.4 \mathrm{~A}$ is due to a beam steering effect ${ }^{15,21,22}$ of the fundamental mode. The increasing contribution of the higher order modes beyond 1.2 A leads to the strong kink around 1.2 A.

Figure 3 illustrates far-field distributions in the lateral direction of the reference $\mathrm{RW}$ laser in $\mathrm{CW}$ mode at $T=20^{\circ} \mathrm{C}$. Starting from the threshold current, the laser demonstrates multi-lateral mode emission across the whole operating current range. This is typical for RW lasers having a ridge as wide as $15 \mu \mathrm{m}$. The laser emits only the fundamental mode along the vertical direction as shown in the inset, which is expected from the concept of the HiBBEE structure. The laser produces $8.5^{\circ} \mathrm{FWHM}$ vertical beam divergence at a drive current of $0.5 \mathrm{~A}$.

The corrugated RW lasers, on the other hand, show a much improved far-field behavior. As illustrated in Fig. 4(a), the corrugations surrounding the ridges discard the higher order lateral modes significantly. Only fundamental mode emission is achieved up to $1.2 \mathrm{~A}$ drive current. Beyond

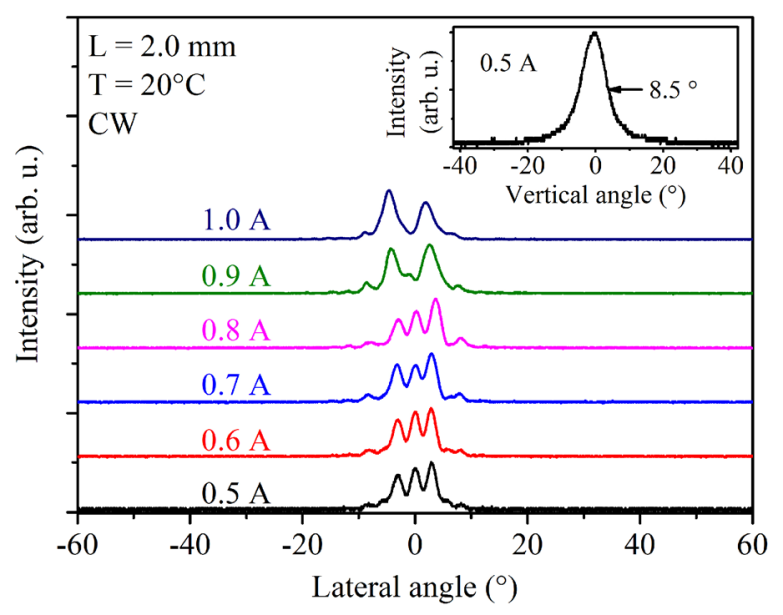

FIG. 3. Far-field distributions in the lateral direction of the reference RW laser presented in Fig. 2 at different drive currents $I$. The inset shows the farfield distribution along the vertical direction at $I=0.5 \mathrm{~A}$. The FWHM beam divergence angle is indicated. The measurements are performed in $\mathrm{CW}$ mode at $T=20^{\circ} \mathrm{C}$.
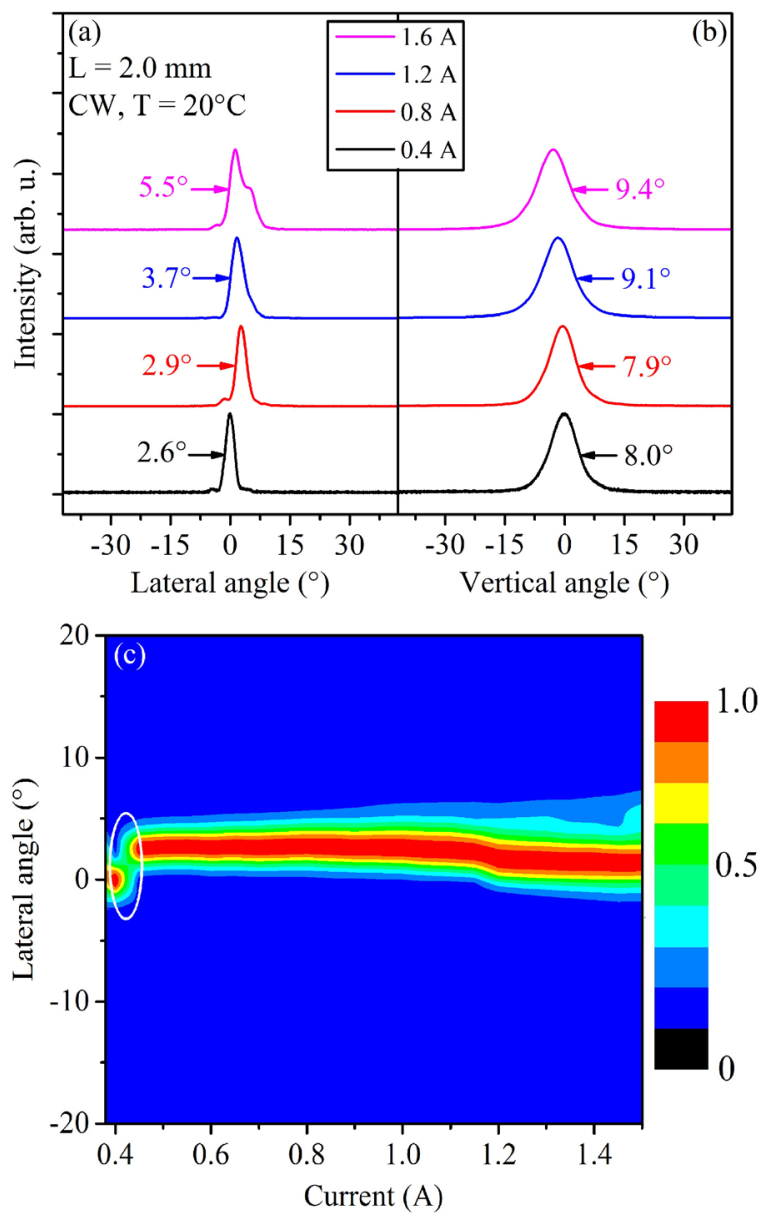

FIG. 4. Far-field distributions in (a) lateral and (b) vertical directions of the corrugated RW laser presented in Fig. 2 at different drive currents. FWHM beam divergence angles are indicated. (c) Color-scale plot of the far-field distributions in the lateral direction as a function of drive current. The lasers have a $2.0 \mathrm{~mm}$ long cavity, and the measurements are performed in $\mathrm{CW}$ mode at $T=20^{\circ} \mathrm{C}$.

1.2 A, the first higher order lateral modes start contributing to the laser emission. A much improved far-field pattern even at the highest drive currents is achieved compared to the reference laser. A change in the propagation direction is visible at $0.4 \mathrm{~A}$, marked by the white circle in Fig. 4(c), which leads to a kink in the output power-current curve [cf. Figs. 4(c) and 2].

Figure 4(b) presents the far-field distributions along the vertical direction at different drive currents. Single mode emission is observed across the whole investigated current range. The broadening of the beam divergence from $2.6^{\circ}$ to $5.5^{\circ}$ in the lateral direction and from $8.0^{\circ}$ to $9.4^{\circ}$ in the vertical direction with increasing drive current from $0.4 \mathrm{~A}$ to 1.6 $\mathrm{A}$ is a consequence of the increased device temperature resulting from Joule heating. The refractive index increases in the RW region with the increase of the device temperature, which results in an increased refractive index contrast between the RW region and its surrounding. This leads to a more confined optical field distribution inside the device producing broader far-field profiles.

The improvement of the beam quality by the corrugated structures is clearly observed by measuring beam quality factors $\mathrm{M}^{2} . \mathrm{M}^{2}$ values are measured using a commercial beam profiler (Spiricon M2-200). The measurement technique 
conforms to the ISO 11146 recommended second moment method. During the measurement, the laser beam was focused to a beam waist by directing the beam through a collimating and a focusing lens. The beam widths are measured at several positions surrounding the beam waist along the beam axis. The measured data points are fitted with the beam waist equation $w^{2}(\mathrm{z})=w^{2}{ }_{0}+\theta^{2}\left(z-Z_{0}\right)^{2}$, where $w, z, Z_{0}$, $w_{0}$, and $\theta$ are the beam width, the distance measured from the focusing lens, the location of the beam waist, the beam width at the beam waist, and the far-field divergence angle, respectively. $\mathrm{M}^{2}$ values are calculated from the fitting parameters $w_{0}, \theta$, and $Z_{0}$ as described in Ref. 23 .

Figure 5 presents $\mathrm{M}^{2}$ factors of the reference and corrugated RW lasers in both lateral and vertical directions. Multilateral mode emission of the reference laser results in $\mathbf{M}^{2}$ factors larger than 4 across the whole investigated drive current range. With increasing drive current, an unexpected reduction of the $\mathrm{M}^{2}$ in the lateral direction is observed, which could be a measurement artifact caused by the second moment method.

In agreement with the measured far-field distributions, the beam quality in the lateral direction is significantly improved with $\mathrm{M}^{2}$ factors being reduced by a factor of $\sim 2$ in the corrugated lasers. $\mathrm{M}^{2}$ values in the lateral direction vary within 2.0-2.3 up to $1.2 \mathrm{~A}$, which are close to the values reported for single-transverse mode lasers at $1060 \mathrm{~nm}$ (Refs. 11 and 15 ) and $980 \mathrm{~nm}^{24}$

$\mathrm{M}^{2}$ values in the vertical directions are improved from a value of $\sim 2.3$ for the reference to $\sim 1.8$ for the corrugated HiBBEE laser. This helpful decrease of the vertical $\mathrm{M}^{2}$ is attributed to the change of the refractive index profile surrounding the RW due to the inhomogeneities of the waveguide. It is a well-known fact that with increasing refractive index contrast between the region of the RW and its surrounding region, the confinements of the vertical modes are also affected. Different distribution of the mode profiles in the vertical direction can induce different amounts of free carrier absorption losses to the fundamental and the higher order modes. Therefore, the mode discrimination can be

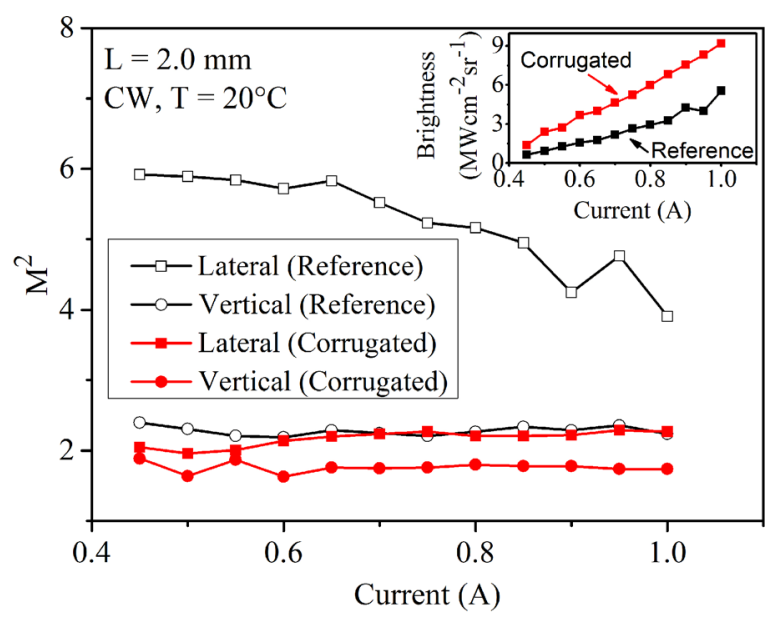

FIG. 5. Lateral (square) and vertical (circle) beam quality factor $\mathrm{M}^{2}$ of the reference RW laser (black, empty symbols) and the corrugated RW laser (red, filled symbols) as a function of drive current in CW mode at $T=20^{\circ} \mathrm{C}$. The inset shows brightness versus drive currents of the lasers. increased which could lead to the observed improvement of $\mathrm{M}^{2}$ in the vertical direction.

Despite a deterioration of the differential quantum efficiency due to increased scattering loss, a significant reduction of the $\mathrm{M}^{2}$ in both lateral and vertical directions of the corrugated laser gives rise to a twice larger brightness as compared to the reference laser, as illustrated in the inset of Fig. 5.

Our proof of the concept of corrugated-RW HiBBEElasers opens an avenue towards achieving much higher brightness than achieved until now. Optimization of the length of the sides of the triangles and their insertion frequency will most probably provide a higher output power and better beam quality and thus improve the brightness. Many other types of corrugations as proposed in Ref. 25 might provide similar or even larger brightness. This work will be done in the future.

In summary, a recent theoretical proposal to use larger horizontal waveguides with higher order mode suppression has been validated. The beam quality of RW lasers guiding multiple-lateral modes is largely improved. The approach is based on enhancing the scattering losses of the higher-order lateral modes by introducing regular repetitive structures along the longitudinal direction of the ridges. Reference RW lasers with $15 \mu \mathrm{m}$ wide homogeneous ridges are found to emit multiple lateral modes. By incorporating triangularshaped corrugations on both sides of the $15 \mu \mathrm{m}$ wide ridges, the higher-order lateral modes can be almost completely suppressed. The corrugated lasers provide a much improved beam quality and two times higher brightness than the reference RW laser counterpart. This concept of introducing corrugations is investigated on a HiBBEE structure, but it will be equally compatible with other laser structures.

The authors are grateful to G. Ropers from FraunhoferInstitut für Nachrichtentechnik for facet coatings.

${ }^{1}$ Y. Inoue and S. Fujikawa, IEEE J. Quantum Electron. 36, 751 (2000).

${ }^{2}$ U. Brauch, P. Loosen, and H. Opower, "High-power diode lasers for direct applications," in High-Power Diode Lasers: Fundamentals, Technology, Applications, edited by R. Diehl (Springer, Berlin, Germany, 2000).

${ }^{3}$ E. Hulicius and V. Kubeček, "Semiconductor lasers for medical applications," in Lasers for Medical Applications: Diagnostics, Therapy and Surgery, edited by H. Jelínková (Woodhead Publishing, Oxford, UK, 2013).

${ }^{4}$ D. M. Roessler, "High-power lasers in materials processing-An automotive perspective," in High Power Lasers-Science and Engineering, NATO ASI Series, Series 3: High Technology Vol. 7, edited by R. Kossowsky, M. Jelinek, and R. F. Walter (Springer, Dordrecht, 1996).

${ }^{5}$ R. W. Lambert, T. Ayling, A. F. Hendry, J. M. Carson, D. A. Barrow, S. McHendry, C. J. Scott, A. McKee, and W. Meredith, J. Lightwave Technol. 24, 956 (2006)

${ }^{6}$ T. Murakami, K. Ohtaki, H. Matsubara, T. Yamawaki, H. Saito, K. Isshiki, Y. Kokubo, A. Shima, H. Kumabe, and W. Susaki, IEEE J. Quantum Electron. 23, 712 (1987).

${ }^{7}$ H. Wenzel, F. Bugge, G. Erbert, R. Hülsewede, R. Staske, and G. Tränkle, Electron. Lett. 37, 1024 (2001).

${ }^{8}$ H. Wenzel, F. Bugge, M. Dallmer, F. Dittmar, J. Fricke, K. H. Hasler, and G. Erbert, IEEE Photonics Technol. Lett. 20, 214 (2008).

${ }^{9}$ T. Kettler, K. Posilovic, L. Ya. Karachinsky, P. Ressel, A. Ginolas, J. Fricke, U. W. Pohl, V. A. Shchukin, N. N. Ledentsov, D. Bimberg, J. Jönsson, M. Weyers, G. Erbert, and G. Tränkle, IEEE J. Sel. Top. Quantum Electron. 15, 901 (2009).

${ }^{10}$ D. Bimberg, K. Posilovic, V. Kalosha, T. Kettler, D. Seidlitz, V. A. Shchukin, N. N. Ledentsov, N. Yu. Gordeev, L. Ya. Karachinsky, I. I. Novikov, M. V. Maximov, Yu. M. Shernyakov, A. V. Chunareva, F. Bugge, and M. Weyers, Proc. SPIE 7616, $76161 \mathrm{I}$ (2010). 
${ }^{11}$ M. J. Miah, T. Kettler, K. Posilovic, V. P. Kalosha, D. Skoczowsky, R. Rosales, D. Bimberg, J. Pohl, and M. Weyers, Appl. Phys. Lett. 105, 151105 (2014).

${ }^{12}$ M. J. Miah, T. Kettler, V. P. Kalosha, K. Posilovic, D. Bimberg, J. Pohl, and M. Weyers, IEEE J. Sel. Top. Quantum Electron. 21, 4900206 (2015).

${ }^{13}$ A. Pietrzak, H. Wenzel, P. Crump, F. Bugge, J. Fricke, M. Spreemann, G. Erbert, and G. Tränkle, IEEE Quantum Electron. 48, 568 (2012).

${ }^{14}$ V. P. Kalosha and D. Bimberg, U.S. patent 9,705,285 (31 January, 2014).

${ }^{15}$ M. J. Miah, V. P. Kalosha, D. Bimberg, J. Pohl, and M. Weyers, Opt. Express 24, 30514 (2016).

${ }^{16}$ Ch. Ribbat, R. L. Sellin, I. Kaiander, F. Hopfer, N. N. Ledentsov, and D. Bimberg, Appl. Phys. Lett. 82, 952 (2003).

${ }^{17}$ E. Gehrig, O. Hess, C. Ribbat, R. L. Sellin, and D. Bimberg, Appl. Phys. Lett. 84, 1650 (2004).
${ }^{18}$ M. T. Kelemen, J. Weber, F. Rinner, J. Rogg, M. Mikulla, and G. Weimann, Proc. SPIE 4947, 252 (2003).

${ }^{19}$ D. Jedrzejczyk, P. Asbahr, M. Pulka, B. Eppich, and K. Paschke, IEEE Photon. Technol. Lett. 26, 845 (2014).

${ }^{20}$ V. P. Kalosha, K. Posilovic, and D. Bimberg, IEEE J. Quantum Electron. 48, 123 (2012).

${ }^{21}$ N. Chinone, J. Appl. Phys. 48, 3237 (1977).

${ }^{22}$ J. Näppi, A. Ovtchinnikov, H. Asonen, P. Savolainen, and M. Pessa, Appl. Phys. Lett. 64, 2203 (1994).

${ }^{23}$ See http://www.ophiropt.com/userfiles/laser/beamprofilers/M2-200s-manual. pdf for "Manual for beam propagation analyzer," M2-200/200s-FW User Guide.

${ }^{24}$ S. Zhao, Y. Wang, H. Qu, Y. Liu, X. Zhou, A. Liu, and W. Zheng, IEEE Photonics Technol. Lett. 29, 2005 (2017).

${ }^{25}$ V. P. Kalosha, K. Posilovic, and D. Bimberg, U.S. patent 8,396,091 (31 January, 2011). 